\title{
Erratum to: Add-On Adefovir Is Superior to a Switch to Entecavir as Rescue Therapy for Lamivudine-Resistant Chronic Hepatitis B
}

\author{
Goh Eun Chung $\cdot$ Won Kim $\cdot$ Kook Lae Lee $\cdot$ Sang Youn Hwang $\cdot$ Jeong-Hoon Lee \\ Hwi Young Kim · Yong Jin Jung • Donghee Kim • Ji Bong Jeong • Byeong Gwan Kim • \\ Yoon Jun Kim $\cdot$ Jung-Hwan Yoon $\cdot$ Hyo-Suk Lee
}

Published online: 2 April 2011

(C) Springer Science+Business Media, LLC 2011

\section{Erratum to: Dig Dis Sci}

DOI 10.1007/s10620-010-1561-2

While describing "Biochemical and Serological Response", values for ETV group (68.8\%) and add-on ADV group (31.3\%) are erroneously published. The correct values are ETV group should be $66.7 \%$ and add-on ADV group should be $38.4 \%$. Corrected text is given below.

Biochemical and Serological Response

Serum ALT levels decreased during the treatment period in both groups (Table 2). However, no significant differences were found between the two groups in the mean reduction and normalization of ALT at week 48. Among $\mathrm{HBeAg}$ positive patients, $\mathrm{HBeAg}$ seroconversion $(66.7 \%$ in the ETV group vs. $38.4 \%$ in the add-on ADV group) and the mean time to $\mathrm{HBeAg}$ seroconversion $(11.83 \pm 8.95$ months in the ETV group vs. $5.40 \pm 1.45$ months in the add-on ADV group) were similar in the two groups $(P=0.229$ and $P=0.190)$.

The online version of the original article can be found under doi:10.1007/s10620-010-1561-2.

G. E. Chung · D. Kim

Department of Internal Medicine, Gangnam Healthcare Center, Seoul National University Hospital, Seoul, Republic of Korea

W. Kim $(\bowtie) \cdot$ K. L. Lee · Y. J. Jung · J. B. Jeong · B. G. Kim Department of Internal Medicine, Seoul Metropolitan Government Seoul National University, Boramae Medical Center 39, Boramae Road, Dongjakgu, Seoul 156-707, Republic of Korea

e-mail: drwon1@snu.ac.kr

S. Y. Hwang · J.-H. Lee $\cdot$ H. Y. Kim · Y. J. Kim · J.-H. Yoon · H.-S. Lee

Department of Internal Medicine and Liver Research Institute, Seoul National University College of Medicine, Seoul,

Republic of Korea 